(C) 2015 IEEE. Personal use of this material is permitted. Permission from IEEE must be obtained for all other uses, in any current or future media, including reprinting/republishing this material for advertising or promotional purposes, creating new collective works, for resale or redistribution to servers or lists, or reuse of any copyrighted component of this work in other works. 


\title{
2015 IEEE RAS Summer School on Agricultural Robotics (SSAR2015)
}

\author{
Robert Fitch, Salah Sukkarieh, Marcel Bergerman, and Eldert van Henten
}

The IEEE RAS Summer School on Agricultural Robotics (SSAR 2015) was held in Sydney, Australia during the southern hemisphere summer, 2-6 February 2015. The school was hosted by the Australian Centre for Field Robotics (ACFR) at The University of Sydney, and was supported in part by the IEEE RAS Technical Education Program. The main aim of the school was to promote robotics research that will enable safe, efficient, and economical production in agriculture and horticulture.

Agricultural robotics is an area of growing interest with the potential to bring about profound economic and social benefits - interest in the area is motivated by the need to improve the industries and processes that feed the planet. Agriculture has historically benefited from a stream of technical innovations, and now must respond to significant new demands and pressures to increase efficiency with limited or decreasing availability of human and environmental resources. In particular, the number of people involved in agriculture and horticulture in at least several major areas of the world has rapidly decreased over the past three decades.

Robotics can play a significant role in improving the efficiency of existing agricultural methods, in introducing fundamentally new methods, and perhaps most importantly in helping to increase the number of people involved in the agricultural sector. Autonomous systems are currently being developed for tasks such as pruning, thinning, harvesting, mowing, targeted spraying, non-chemical weed control, and high-resolution crop surveillance. A new cohort of people in agriculture will develop, commercialise, and operate such systems.

SSAR2015 was endorsed by the RAS Technical Committee on Agricultural Robotics and Automation and was conceived as an educational event that would both collect and disseminate technical information and help to foster a cohesive and collaborative research community. The program consisted of presentations, hands-on activities, a farm visit, a robot demonstration, and social events. The school was officially opened by the New South Wales (state) Minister for Primary Industries, Katrina Hodgkinson.

There were 18 presentations given by speakers spanning six different countries. The backgrounds of the speakers are diverse, ranging from roboticists to government and industry leaders (Figure 1). Technical lectures covered topics in sensing and perception, planning and control, manipulation and grasping, and systems engineering in an agricultural context. We also heard about major projects in the US, UK, Europe, Australia, and Pakistan. Government and industry leaders spoke about industry-specific challenges, including a presentation by Daniel Schmoldt from the US Department of Agriculture.

Hands-on activities consisted of lab-based exercises in estimation and navigation (using data from a corn field), and in perception algorithms for plant detection and classification from camera images (Figure 2). We also visited an operational robotic rotary milking system installation at The University of Sydney's Camden campus (Figure 3). On the final day of the school, we toured the ACFR's field lab and had a demonstration of the ACFR's Ladybird robot designed for operations in vegetable crops (Figure 4).

Social events played an important part in the success of the school. On the first day, participants introduced themselves via a lightning round that consisted of a rapid sequence of single-slide, 60-second presentations. A participant poster session followed, promoting informal discussion in a semistructured environment. This introductory session set the stage for further discussion throughout the week during coffee breaks, a group dinner, and the closing reception.

The 40 student participants came from a mix of backgrounds and countries. In addition to robotics graduate students and researchers, there were many participants new to IEEE. A group photo is shown in Figure 5.

One of the main outcomes of the school aside from technical education was indeed to create a new feeling of community among the variety of participants. There is now a clear desire to meet again through future summer schools and potentially new specialised conferences and workshops.

The school received considerable attention from Australian and international media. Several journalists attended selected presentations and interviewed speakers, resulting in multiple articles in print and online. Representative examples include: http://www.abc.net.au/news/201502-04/agricultural-robotics-future-jobs/6068450/,

http://www.abc.net.au/news/2015-02-10/nsw-robotchicken-collecting-free-range-eggs/6083152/, and http://www.abc.net.au/news/2015-02-12/robotics-foragriculture-leave-large-machinery-behind/6088112/.

SSAR2015 was organised by Robert Fitch (ACFR), Salah Sukkarieh (ACFR), Marcel Bergerman (CMU), and Eldert van Henten (Wageningen University). We would like to thank all participants, speakers, and volunteers for their help. Special thanks go to Ruth Olip, Claire Devonport, and Dannielle Williams for superb local arrangements and administration, and to the IEEE RAS Technical Education Program for its generous support. More information about the school can be found on its website, http://www.acfr.usyd.edu.au/education/ssar2015.shtml. 


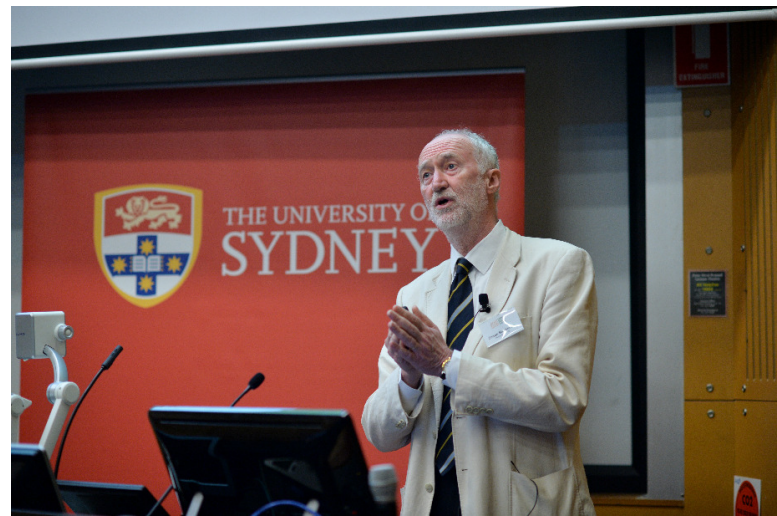

(a)

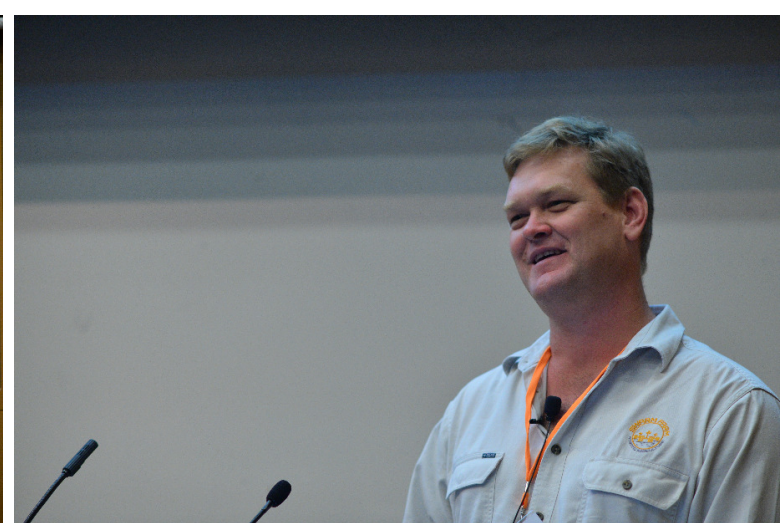

(b)

Fig. 1. Speakers came from a variety of backgrounds. (a) Simon Blackmore (Harper Adams University, UK) talks about successes, motivation, and challenges in agricultural robotics. (b) Andrew Bate (SwarmFarm Robotics), a farmer-turned-robotics entrepreneur, talks about commercialising a multirobot system for broadacre agriculture.

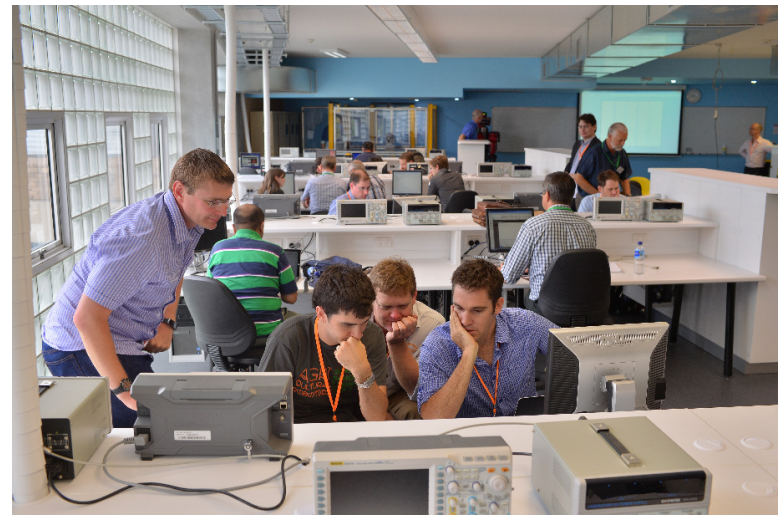

(a)

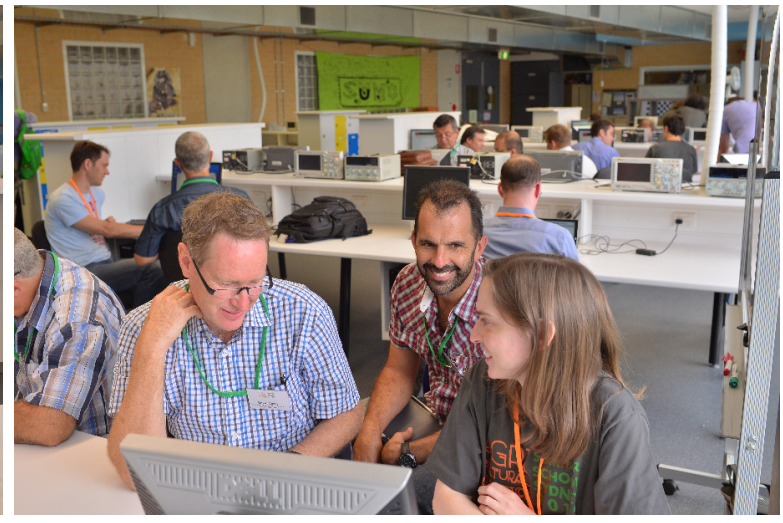

(b)

Fig. 2. (a) Attendees participating in hands-on activities with algorithms for estimation and navigation among corn rows. (b) New friendships formed within working groups.

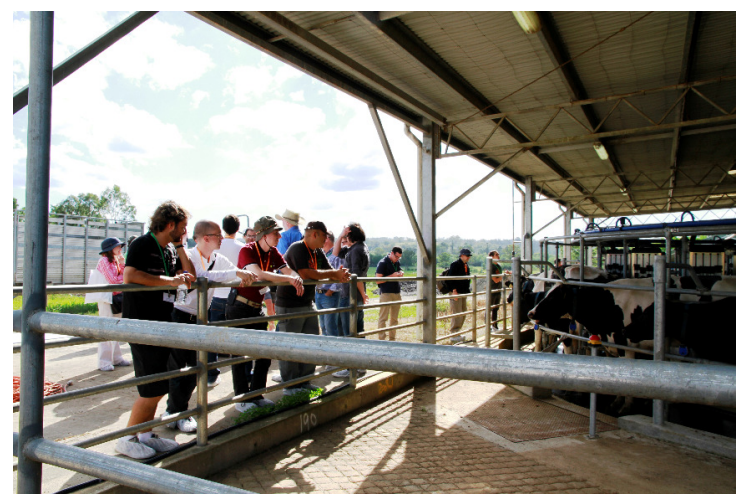

Fig. 3. Participants visited Camden, NSW to view a demonstration of an operational robotic rotary milking system.

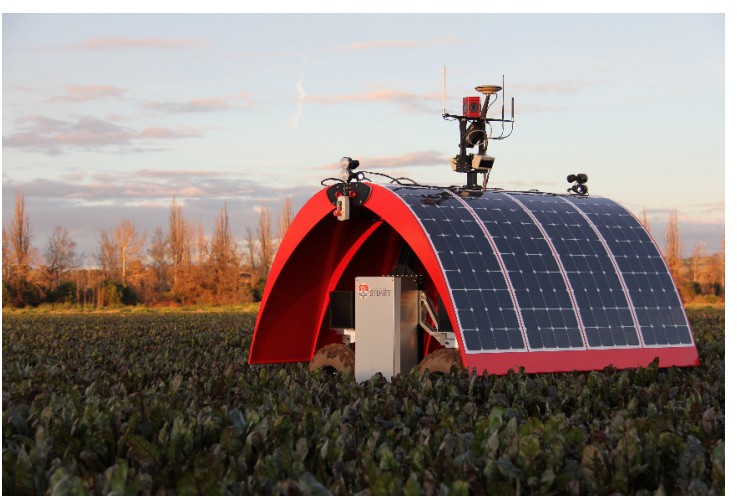

Fig. 4. The Ladybird robot, designed for operations in vegetable crops, shown here at a field trial in Cowra, NSW. 


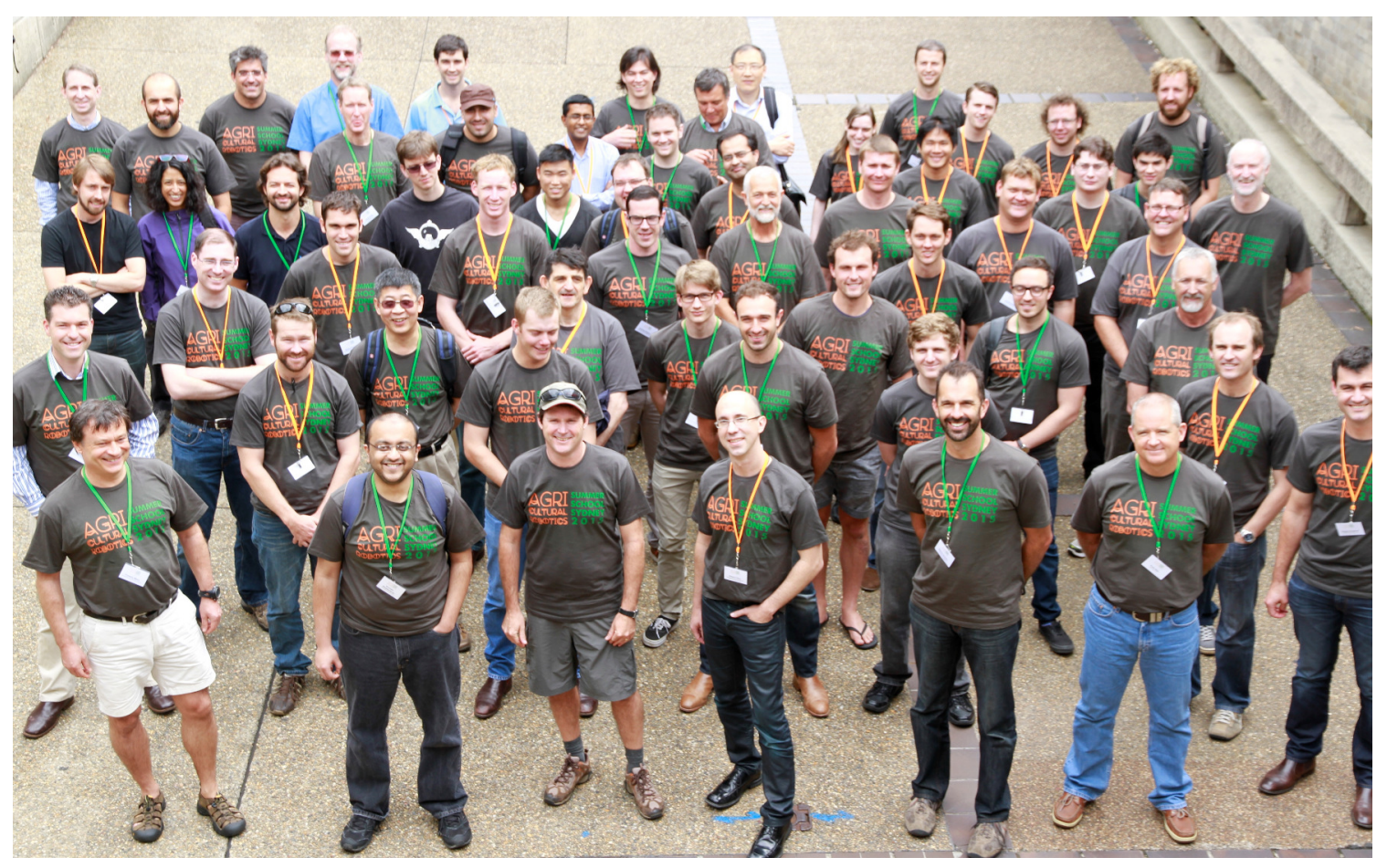

Fig. 5. SSAR2015 participants. 